La revue La revue pour l'histoire du CNRS

POUR LHISTORE DU CNRS $16 \mid 2007$

L'expertise scientifique

\title{
Les années Joliot
}

La naissance du Laboratoire de physique nucléaire d'Orsay (1956-1958)

René Bimbot

\section{CpenEdition}

Journals

Édition électronique

URL : https://journals.openedition.org/histoire-cnrs/1597

DOI : 10.4000/histoire-cnrs. 1597

ISSN : 1955-2408

Éditeur

CNRS Éditions

Édition imprimée

Date de publication : 3 avril 2007

ISBN : 978-2-271-06453-0

ISSN : $1298-9800$

Référence électronique

René Bimbot, "Les années Joliot », La revue pour I'histoire du CNRS [En ligne], 16 | 2007, mis en ligne le 26 mars 2009, consulté le 20 mai 2021. URL : http://journals.openedition.org/histoire-cnrs/1597 ;

DOI : https://doi.org/10.4000/histoire-cnrs. 1597

Ce document a été généré automatiquement le 20 mai 2021.

Comité pour l'histoire du CNRS 


\title{
Les années Joliot
}

\author{
La naissance du Laboratoire de physique nucléaire d'Orsay (1956-1958)
}

\section{René Bimbot}

La création du Laboratoire de physique nucléaire

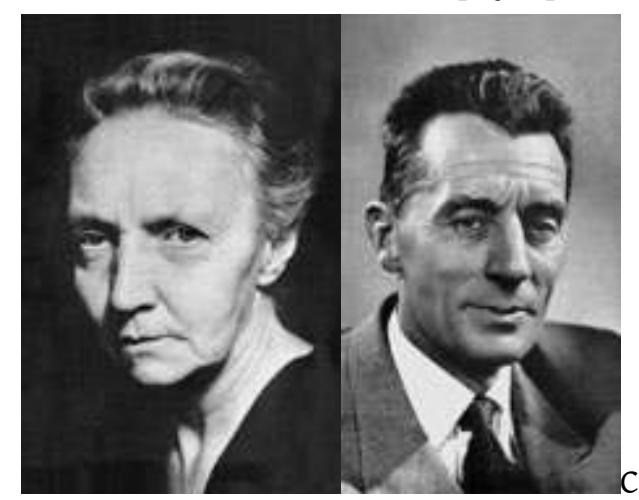

C'est sans conteste à Irène Joliot-Curie que l'on doit la création du laboratoire qui deviendra l'Institut de physique nucléaire d'Orsay [1]. En effet, dès $1942{ }^{1}$, Irène est à la recherche d'un grand terrain, dans la banlieue sud de Paris. Suffisamment proche de la ligne de Sceaux, celui-ci permettrait une extension des laboratoires de physique nucléaire, trop à l'étroit dans leurs locaux parisiens pour envisager la construction des grands instruments désormais nécessaires au développement de cette discipline. Mais c'est bien après la fin de la guerre, en 1954, que ce projet va se concrétiser. Cette année-là vient en discussion au Parlement la question de la participation française au CERN, centre de recherche nucléaire où des moyens doivent être mis en commun à l'échelle européenne. Frédéric et Irène Joliot sont consultés sur ce sujet et défendent la position selon laquelle cette participation ne peut avoir de sens que si la France dispose en parallèle de centres universitaires bien équipés, notamment en accélérateurs de particules, ce qui n'est vraiment pas le cas à cette époque ${ }^{2}$. En effet, la plus puissante machine dont disposent les chercheurs français est le cyclotron* du Collège de France, de diamètre égal à 85 centimètres, qui accélère des protons jusqu'à $13 \mathrm{MeV}^{3}$ et des deutons jusqu'à $6,7 \mathrm{MeV}$, alors que de grands accélérateurs existent déjà dans le monde. À Berkeley, par exemple, un synchrocyclotron* de 184 inches (4,60 mètres) accélère des protons jusqu'à $400 \mathrm{MeV}$ 
depuis 1946, et un synchrotron* à protons de $6 \mathrm{GeV}(6000 \mathrm{MeV})$, le bévatron, vient d'être mis en service.

1 À cette époque, les principaux problèmes que la physique nucléaire tente de résoudre sont relatifs à la structure des noyaux, c'est-à-dire à l'organisation des nucléons protons et neutrons - qui les constituent, à l'origine des forces nucléaires, qui lient ces nucléons entre eux, et au mécanisme des réactions nucléaires ${ }^{4}$. En ce qui concerne ces projectiles - principalement des protons -, on distingue trois domaines d'énergie :

- Les faibles énergies (inférieures à $20 \mathrm{MeV}$ ), sont couvertes par les accélérateurs électrostatiques* et les petits cyclotrons, comme celui du Collège de France. À ces énergies, les projectiles peuvent « exciter » les noyaux cibles - c'est-à-dire leur communiquer une certaine quantité d'énergie - , ou les transformer en d'autres noyaux, généralement radioactifs, assez proches des noyaux cibles. Les noyaux excités et les isotopes radioactifs ainsi produits constituent des sources d'information importantes sur la structure nucléaire.

- Les moyennes énergies, de l'ordre de la centaine de $\mathrm{MeV}$, donnent accès à des réactions nucléaires de caractéristiques différentes, conduisant notamment à l'émission de nombreux nucléons, et à une grande variété d'isotopes radioactifs.

- Enfin, les collisions se produisant aux hautes énergies - supérieures à $300 \mathrm{MeV}$ - peuvent engendrer de nouvelles particules, tels les mésons, que l'on soupçonne de jouer un rôle dans la transmission des forces nucléaires.

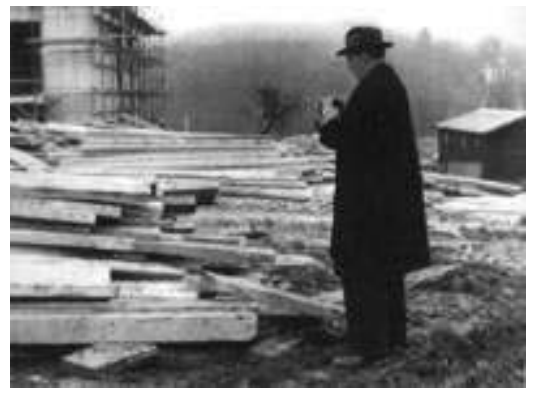

Frédéric Joliot visitant le chantier du laboratoire (hiver 1957-1958). @ IPN Orsay

2 La requête des Joliot est finalement acceptée et il est décidé de créer en région parisienne deux laboratoires universitaires de physique nucléaire, équipés, l'un d'un synchrocyclotron à protons de $150 \mathrm{MeV}$, l'autre d'un accélérateur linéaire* d'électrons d'énergie égale à $1,3 \mathrm{GeV}$. Avec le synchrotron à protons de $3 \mathrm{GeV}$ - le futur Saturne qui sera construit à Saclay, ces grands accélérateurs constitueront les outils nécessaires pour que la physique nucléaire française reprenne une place honorable dans la recherche internationale, sinon celle, de premier plan, qui était la sienne avant le conflit mondial.

3 Ces nouveaux laboratoires seront implantés sur un vaste terrain, de 150 ha, disponible sur les communes de Bures-sur-Yvette et Orsay. Le premier sera le Laboratoire de physique nucléaire, une extension de l'Institut du radium dont Irène Joliot-Curie assure la direction depuis 1946. Le synchrocyclotron qui a été choisi pour l'équiper orientera nettement ses activités vers la physique à moyenne énergie. Des expériences pourront être également poursuivies à faible énergie grâce à de petites machines électrostatiques dont il est prévu d'équiper aussi le laboratoire. Le second, le Laboratoire de l'accélérateur linéaire (LAL), plutôt tourné vers les hautes énergies - tout comme le synchrotron de Saclay - sera pris en charge par les chercheurs de l'École normale supérieure, sous la responsabilité d'Yves Rocard ${ }^{5}$. Ces deux centres de recherche 
constituent le noyau de la Faculté des Sciences d'Orsay, qui deviendra plus tard l'université Paris-Sud-XI [3].

4 Le feu vert des autorités et les crédits ayant été obtenus, la construction du Laboratoire de physique nucléaire va commencer très vite. Sous la responsabilité administrative de M. Surleau, commissaire à la construction de la toute nouvelle Faculté, Robert Bouchez, Michel Riou et Jean Teillac, trois enseignants de l'Institut du radium, élaborent le programme de construction et supervisent les plans des architectes. Ils sont bientôt assistés d'autres chercheurs, ainsi que d'ingénieurs de haut niveau qui ont pu être embauchés grâce à la constitution d'un nouveau cadre universitaire, celui des contractuels de physique nucléaire. Cette innovation administrative autorise des rémunérations comparables à celles des personnels du CEA, elles-mêmes bien supérieures à celles des agents du CNRS. Les plans du nouveau laboratoire sont terminés en 1955 et les bâtiments commencent à sortir de terre en 1956.

5 Malheureusement, l'instigatrice de cet ambitieux projet, Irène Joliot-Curie, ne verra pas son achèvement. Elle meurt le 17 mars 1956, minée par les nombreux rayonnements qu'elle a reçus, d'abord lorsqu'elle assistait sa mère dans sa mission de radiographie des blessés sur le front de la Grande Guerre, puis au cours de près de quarante années de recherche mettant en œuvre de puissantes sources radioactives. C'est Frédéric Joliot qui lui succèdera comme titulaire de la chaire de Physique nucléaire et Radioactivité, donc comme directeur de l'Institut du radium et ipso facto du Laboratoire d'Orsay.

6 C'est donc sous sa direction que s'achèvera la construction du synchrocyclotron, qui, familièrement appelé le "Synchro ", constituera la pièce maîtresse du laboratoire pendant ses premières années de fonctionnement $[4,5]$.

Les premiers chercheurs du laboratoire d'Orsay

7 Comme nous l'avons déjà mentionné, c'est de l'Institut du radium qu'était partie l'idée d'une extension hors de Paris, et c'étaient les chercheurs de cet institut qui avaient défini les caractéristiques du synchrocyclotron. Paradoxalement, le Laboratoire de physique et chimie nucléaires du Collège de France, dirigé par Frédéric Joliot, n'avait pas été impliqué dans le projet, malgré les liens plus qu'étroits qui unissaient les directeurs de ces deux unités. Mais rapidement, certains membres de ce laboratoire du Collège, et en particulier les jeunes docteurs, avaient pensé que cette nouvelle machine leur offrirait des possibilités bien supérieures à celles de leur petit cyclotron, et souhaité être associés à la définition des programmes de recherche.

8 Lorsque Frédéric Joliot, succédant à Irène à la direction du laboratoire d'Orsay, décida d'y faire transférer le cyclotron du Collège de France, le nombre de ces personnes demandant leur rattachement à Orsay se trouva encore renforcé. Parmi elles figuraient onze théoriciens, dont quatre étudiants qui, sous l'impulsion de Maurice Jean, fondèrent le groupe de physique théorique du laboratoire. Quelques chercheurs en provenance du laboratoire de synthèse atomique d'Ivry-sur-Seine rejoignirent également Orsay.

9 Le cyclotron de Joliot est transporté à Orsay en avril 1958, et installé dans une petite extension du bâtiment du synchrocyclotron, qui porte le numéro 101. Le bâtiment ${ }^{6} 102$, tout proche, accueillera l'ensemble du personnel qui, toutes catégories confondues (chercheurs, ingénieurs, techniciens et administratifs), s'élève à environ 150 personnes à la mi 1957, et atteindra le chiffre de 250 l'année suivante. Dans ce bâtiment, sous la direction de René Bernas, sera construit un séparateur d'isotopes, équipement lourd 
venant en complément du synchrocyclotron pour l'étude des nouveaux noyaux qui seront synthétisés par réactions nucléaires.

Non loin de cet ensemble, qui constitue le cœur du Laboratoire de physique nucléaire, se construit le bâtiment 104, qui accueillera le Laboratoire de spectroscopie dirigé par Salomon Rosenblum, en provenance de Bellevue. Ce laboratoire, noyau de l'actuel CSNSM 7 , restera distinct du futur Institut de physique nucléaire, mais des collaborations étroites auront souvent lieu entre eux.

La mort de Frédéric Joliot

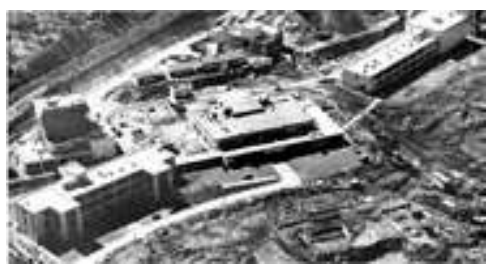

Les premiers bâtiments d'Orsay (1958). Au premier plan, à gauche, se tient le bâtiment 102 (direction, physiciens, séparateur d'isotopes...) et, juste derrière lui, la tour des hautes tensions, en construction. Au centre se trouve le bâtiment du synchrocyclotron, et en haut à droite le laboratoire de spectroscopie.@ IPN Orsay

11 Les premiers essais de production d'un faisceau interne dans le synchrocyclotron ont lieu au début du mois de juin 1958. En mesurant l'intensité du courant capté par une cible de mesure placée à l'intérieur de la machine, les ingénieurs décèlent la présence $\mathrm{du}$ faisceau jusqu'au rayon maximum de la machine. Afin de corroborer ces observations, et malgré le danger que représente une telle initiative, Frédéric Joliot, qui a suivi de très près la construction de la machine, se fait enfermer dans la salle du faisceau, un détecteur de rayonnement à la main... Quelques minutes plus tard, il demande à sortir. Il a effectivement constaté une considérable augmentation de la radioactivité ambiante lorsque le faisceau est accéléré !

Mais la joie provoquée par ce succès au sein du Laboratoire de physique nucléaire sera de courte durée. Deux mois plus tard, le 14 août 1958, ses membres apprennent, en même temps que la France entière, la nouvelle du décès de Frédéric Joliot, dont la santé s'était beaucoup dégradée depuis la perte de son épouse. Avant même que les premières expériences aient commencé sur sa nouvelle machine, le Laboratoire de physique nucléaire est doublement orphelin. Ses deux prestigieux fondateurs ont disparu en l'espace de deux ans !

13 Le laboratoire, dont la plupart des membres proviennent des laboratoires des JoliotCurie, subira ce double deuil de plein fouet. Pendant de nombreuses années, les nouveaux chercheurs qui viendront y travailler, ainsi que tous les visiteurs, seront impressionnés par la profusion de photographies du couple de savants, affichées dans la plupart des bureaux et autres locaux.

14 Les années qui suivront verront, sous la direction de Jean Teillac, la montée en puissance des activités du laboratoire, qui prendra le nom de «Laboratoire Joliot-Curie de physique nucléaire ». L'arrivée de nombreux jeunes, techniciens, ingénieurs et chercheurs, l'intérêt des travaux scientifiques et techniques entrepris, et les succès qui en découleront viendront atténuer ce sentiment de deuil et relancer une véritable dynamique de recherche.

15 Vers la fin des années 1960, un monument à la mémoire de ses fondateurs sera érigé entre les deux bâtiments principaux du laboratoire. 
La « Big Science » en marche!

16 Ainsi, après avoir atteint les États-Unis dans les années 1940, la science des grands instruments, la «Big Science ", s'installait en France en commençant par Orsay et Saclay. Les physiciens allaient devoir passer d'une recherche artisanale à des techniques quasi-industrielles utilisant des équipements très coûteux. Frédéric Joliot en était bien conscient. Dans la conclusion, restée très actuelle, de l'un de ses derniers articles [6], il exprimait ses craintes au sujet des dangers que ces nouvelles conditions pouvaient présenter pour la créativité des chercheurs :

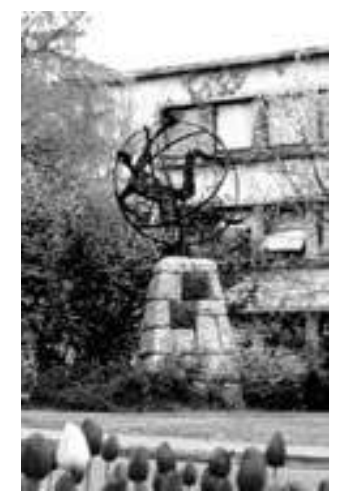

Monument dédié à la mémoire de Frédéric Joliot et Irène Joliot-Curie, réalisé par le sculpteur Robert Couturier et exposé entre les deux bâtiments principaux de l'Institut de physique nucléaire en 1967. (C) IPN Orsay

17 « Dans cette transition de l'échelle artisanale à l'échelle industrielle, il me semble indispensable d'être conscient de ces dangers et de trouver les conditions d'utilisation de l'équipement qui n'étoufferont pas la personnalité du chercheur. On ne peut faire œuvre originale à la chaîne. »

\section{BIBLIOGRAPHIE}

[1] L'IPN d'Orsay, 50 ans de recherche. R. Bimbot, La revue pour l'histoire du CNRS, $n^{\circ} 15$, novembre 2006, p. 30.

[2] Irène Joliot-Curie et la création de l'Institut de physique nucléaire. Plaquette réalisée par l'Institut de physique nucléaire d'Orsay, Université Paris-Sud, 1976.

[3] La recherche et l'enseignement en physique nucléaire au Centre d'Orsay, J. Teillac,

L'âge nucléaire, $\mathrm{n}^{\circ} 12$, septembre-octobre 1958, p. 241.

[4] L'équipement des laboratoires en physique nucléaire au Centre d'Orsay. M. Riou,

L'âge nucléaire, $\mathrm{n}^{\circ} 12$, septembre-octobre 1958, p. 245.

[5] Le synchrocyclotron d'Orsay. C. Bergamaschi et P. Radvanyi, La Nature, 3292, août 1959, p. 337.

[6] Le nouveau centre de recherches fondamentales en physique nucléaire d'Orsay et 
la formation des chercheurs. F. Joliot, L'âge nucléaire, n¹1, juillet-août 1958, p. 183.

\section{NOTES}

1.Comme l'atteste une lettre qu'elle adresse au recteur de l'université de Paris (référence bibliographique [2]).

2.Ces centres de recherche devaient constituer, avec le CERN, le volet fondamental de la recherche nucléaire française. Le Commissariat à l'énergie atomique, qui avait été créé dès 1945, en assurait, pour sa part, les applications pacifiques et militaires, ce qui ne le dispensait d'ailleurs pas de développer, lui aussi, des recherches fondamentales servant d'assise à ces recherches appliquées.

3.1 MeV (1 million d'électron-volt) est l'énergie acquise par un électron (ou par un proton) accéléré par une tension de 1 million de volts.

4.Les réactions nucléaires sont des modifications des noyaux engendrées par des collisions avec des projectiles accélérés.

5.Son premier directeur sera Hans Halban, qui avait participé, en 1940, avec Lew Kowarski et Frédéric Joliot aux expériences sur la mise en évidence de la possibilité de réactions en chaîne lors de la fission de l'uranium.

6.L'ensemble des bâtiments constituant le Laboratoire de physique nucléaire, numérotés à partir de 100, constituent le groupe 1, alors qu'à moins d'un kilomètre de là, ceux du groupe de l'accélérateur linéaire, le groupe 2 , sont numérotés à partir de 200. C'est à partir de cette base que sont encore référencés tous les bâtiments de l'université d'Orsay.

7.Centre de spectrométrie nucléaire et de spectrométrie de masse.

\section{RÉSUMÉS}

Avec la construction d'un synchrocyclotron à protons, la création du Laboratoire de physique nucléaire d'Orsay, extension de l'Institut du radium de Paris, marque l'entrée des grands accélérateurs dans la recherche française. Mais les débuts du premier laboratoire de la future université d'Orsay vont être endeuillés par la disparition de ses deux fondateurs, Irène JoliotCurie en 1956, et Frédéric Joliot en 1958.

The Orsay Laboratoire de physique nucléaire (Nuclear Physics Laboratory) was founded as an extension of the Paris Institut du radium (Radium Institute) and equipped with a proton synchrocyclotron, the first of the French big accelerators. But the birth of this new laboratory, at the origin of the future Orsay University, was plunged into mourning by the death of its two founders, Irène Joliot-Curie in 1956 and Frédéric Joliot in 1958. 


\section{AUTEUR}

\section{RENÉ BIMBOT}

René Bimbot est agrégé de physique, docteur ès sciences, directeur de recherche émérite à l'IPN et au CNRS. 